\title{
Detection of GPS signal scattered from mountain
}

\author{
Yoshiya Arakaki ${ }^{\mathrm{a})}$ and Jun Amagai \\ Okinawa Electromagnetic Technology Center, National Institute of Information \\ and Communications Technology (NICT), \\ 4484 Onna, Onna-son, Kunigami-gun, Okinawa 904-0411, Japan \\ a)arakaki@nict.go.jp
}

\begin{abstract}
We succeeded in receiving the scattered signal of the GPS from point other than the specular reflection point using beam antenna. Characteristic echoes with Doppler frequency shift dependent on range were derived from the observed data with integration period of 40 seconds.
\end{abstract}

Keywords: radar, passive radar, GNSS

Classification: Sensing

\section{References}

[1] M. Martin-Neira, "A passive reflectometry and interferometry system (PARIS): Application to ocean altimetry," ESA J., vol. 17, pp. 331-355, 1993.

[2] T. Hobiger, J. Amagai, M. Aida, and H. Narita, "A real-time GNSS-R system based on software-defined radio and graphics processing units," Adv. Space Res., vol. 49, no. 7, pp. 1180-1190, 2012. DOI:10.1016/j.asr.2012.01.009

[3] IS-GPS-200, Navstar GPS space segment/Navigation User Segment Interfaces, 9 Dec. 2015.

[4] IS-GPS-705, Navstar GPS space segment/User Segment L5 Interfaces, 24 Sep. 2013.

\section{Introduction}

While Global Navigation Satellite system (GNSS) signals specularly reflected by ground or ocean surface have been successfully detected using a technique GNSSreflectometry (GNSS-R) [1, 2], GNSS signals scattered from other than the specular reflection point are very hard to be detected because the detected power is too small.

Introducing beam antenna and longer integration period to detect such a weak signal, we successfully detected scattered signal from mountain $3 \mathrm{~km}$ away.

\section{Observation system}

\subsection{Hardware}

Power flux-density illuminated by GNSS at the surface of the Earth is around $0.1 \mathrm{pW} / \mathrm{m}^{2}$ and too weak to get sufficient signal to noise ratio $(S N R)$ for detecting scatter from target object. The $S N R$ is generally defined by following equation. 


$$
S N R=\sqrt{\frac{S}{N}} \cdot \sqrt{2 B T}
$$

Where $S$ is power of the signal, $N$ is power of noise, $B$ is signal bandwidth and $T$ is integration time. According to this equation, we can say that it is necessary to increase $S$ and/or $T$ to get sufficient $S N R$.

To increase $S$, we adopted an offset parabolic antenna with a diameter of $75 \mathrm{~cm}$ that was originally designed for receiving broadcasting satellite signal. We removed feed horn from the antenna and mounted omnidirectional antenna for GNSS at the focus of the parabola instead of the horn. Although a ordinarily GNSS antenna is designed to detect right-handed circularly polarization, by reflecting it on the parabolic surface, it becomes an antenna with the opposite polarization suitable for receiving scattered signal.

The signal received by the antenna was converted to an IF signal centered at $80 \mathrm{MHz}$ with a bandwidth of $32 \mathrm{MHz}$ and undersampled by an AD converter at a sample rate of $64 \mathrm{Msps}$. Signal received by ordinary omnidirectional antenna was also processed with the same manner as that for scattered signal and utilized for phase compensation for L5 signal. The sampled signals are transferred to a computer and analyzed in it.

\subsection{Signal analysis}

Recently so many types of navigation signals are transmitted from GNSS. First, we tried to use L2CL signal of GPS [3] because L2CL signal has no overlay data and is suitable for long coherent integration. However, since the chip rate of L2CL signal is $1,023 \mathrm{kcps}$, only low range resolution can be obtained. Therefore, after confirming that the scatter signal was successfully received using the L2CL signal, the L5 signal with a high chip rate of $10,230 \mathrm{kcps}$ [4] was used to obtain higher range resolution. Hereinafter, the case of the L5 signal will be described.

The sampled signal is correlated with the replica signal every code length of $1 \mathrm{~ms}$. The complex values obtained as a result of the correlation processing were coherently integrated over $20 \mathrm{~ms}$ referring the phase of the overlay data of the reference signal received by the omnidirectional antenna. Furthermore, the integrated values were corrected using the delay and phase of the direct signal. We generated a range-Doppler map using the integrated values for 40 seconds.

\section{Delay and Doppler frequency shift}

The actual distance $|\vec{R}|$ between the receiving point and the scattering point can be calculated from the apparent distance $r$ obtained from the arrival time difference using the following well-known equation.

$$
|\vec{R}|=\frac{\frac{1}{2} r^{2}+r|\vec{S}|}{(|\vec{S}|+r)-|\vec{S}| \cos \Delta \theta}
$$

Where $\vec{S}$ is a vector from the receiving point to the satellite and $\Delta \theta$ is the angle between the satellite and the scattering point as seen from the receiving point. 
Let $E l$ be the elevation angle of the satellite, and $\triangle A z$ be the azimuth angle difference between the satellite and the scattering point as seen from the receiving point, $\cos \Delta \theta$ is expressed by $\cos E l \cos \Delta A z$. Since $|\vec{S}|$ is much larger than $r$, the above equation is simplified as follows.

$$
|\vec{R}| \approx \frac{r}{1-\cos E l \cos \triangle A z}
$$

The delay change rate $\dot{\tau}$ can be obtained by the following equation.

$$
\begin{aligned}
\dot{\tau} & =\frac{1}{c} \frac{d}{d t}\left\{\left(\left|\vec{S}^{\prime}\right|+|\vec{R}|\right)-|\vec{S}|\right\} \\
& =\frac{1}{c} \frac{d}{d t}\left|\overrightarrow{S^{\prime}}\right|-\frac{1}{c} \frac{d}{d t}|\vec{S}|
\end{aligned}
$$

Where $\vec{S}^{\prime}$ is a vector from scattering point to the satellite and $c$ is the speed of light. Therefore, the Doppler frequency shift $\Delta f$ is obtained by the following equation using wevelength of the signal $\lambda$.

$$
\Delta f=\frac{1}{\lambda} \frac{d}{d t}|\vec{S}|-\frac{1}{\lambda} \frac{d}{d t}\left|\vec{S}^{\prime}\right|
$$

Let $\vec{V}$ be the velocity vector of the satellite in the ground fixed coordinate system, the Doppler frequency shift is rewritten as follows.

$$
\Delta f=\frac{1}{\lambda}\left\{\frac{\vec{S}}{|\vec{S}|}-\frac{\overrightarrow{S^{\prime}}}{\left|\overrightarrow{S^{\prime}}\right|}\right\} \cdot \vec{V}
$$

\section{Experimental results}

The view of the target mountain (Mt. Onna, 26 $26^{\prime} 44^{\prime \prime} \mathrm{N}, 127^{\circ} 52$ ' 29" E, Altitude $364 \mathrm{~m})$ from the receiving site $\left(26^{\circ} 29^{\prime} 55^{\prime \prime} \mathrm{N}, 127^{\circ} 50^{\prime} 41^{\prime \prime} \mathrm{E}\right.$, Altitude $18 \mathrm{~m}$ ) is shown in Fig. 1. The beam of the $75 \mathrm{~cm}$ parabolic antenna is oriented straight toward the Mt. Onna. It is estimated that this antenna has a gain of around $17 \mathrm{~dB}$ and a half power beamwidth (HPBW) of around 20 degrees for both L2 and L5 bands.

A range-Doppler map obtained by analyzing SV \#32 is shown in Fig. 2 as an example. The abscissa in this figure is the pseudo-range before applying the correction indicated by Equation (3). In this case, the angle difference $\Delta \theta$ between the satellite and the scattering point as seen from the receiving point is sufficiently large (opposite direction), in other words, the direction of the scattering point is far away from the specular reflection point. It can be seen that the Doppler frequency shift of a signal considered to be a scattered echo increases with distance.

In the L5 band, five satellites were received at the observation time (UT 2:23 on April 28, 2017), and scattered echoes were detected by all of these satellites. Likewise, eight satellites were received in the L2 band, and scatted echoes were detected in all of these satellites as well (UT 07:38 on December 19, 2016).

The normalized correlation amplitude is about $4 \times 10^{-4}-7 \times 10^{-4}$ in the L2 band and less than $2 \times 10^{-4}$ in the $\mathrm{L} 5$ band. This is probably because the range resolution of the L5 band are higher than the L2 Band, so the volume contributing to scattering is relatively small. 


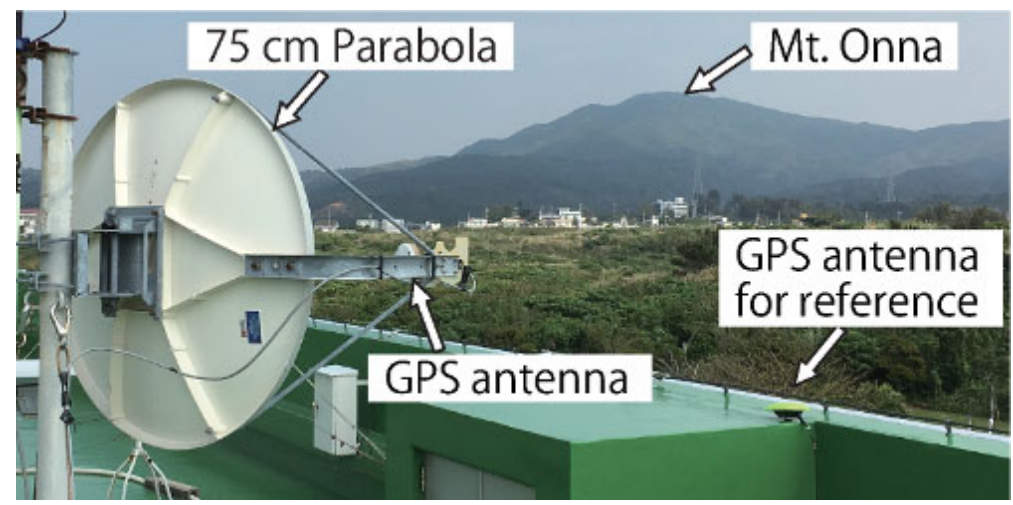

Fig. 1. Outdoor equipment for the experiment and Mt. Onna that causes scattering.

gps_17118-a17032_1_1_ddpa.corr $:$ int $=39.98$ s, c_int $=40.00$ s, no surn

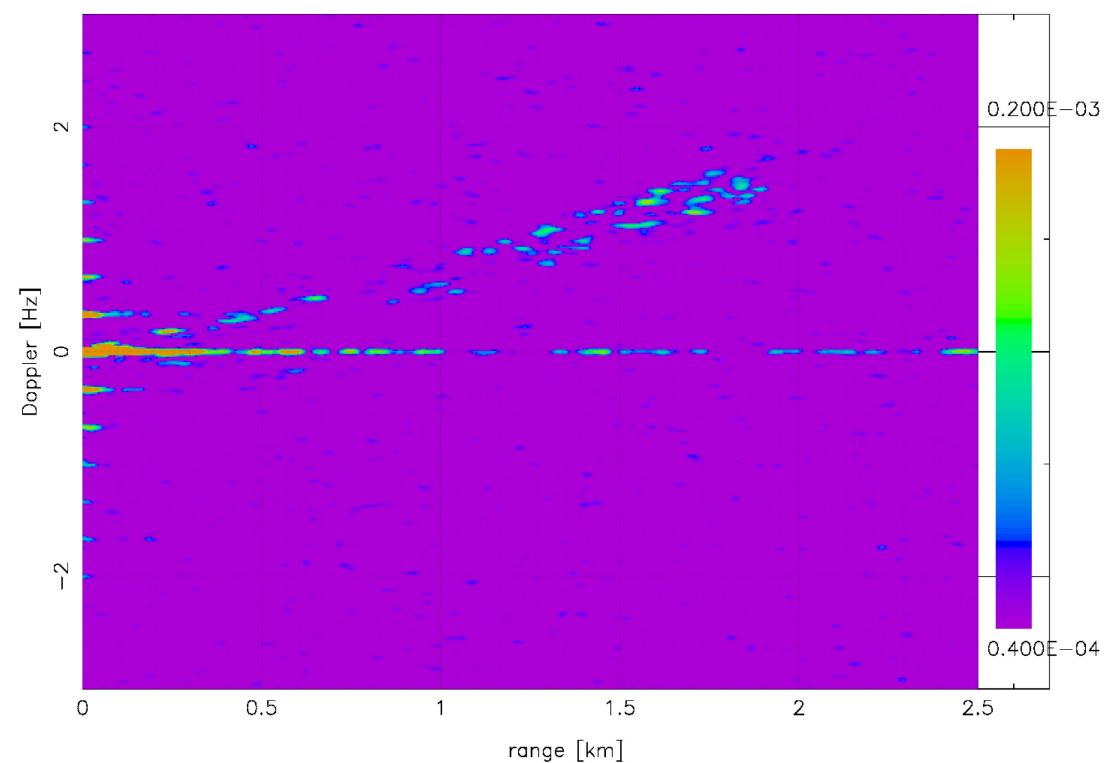

Fig. 2. Range-Doppler map of the echoes from the mountain obtained by analyzing observation data. The normalized correlation amplitude is indicated by color.

Fig. 3 shows the range-Doppler map calculated using equation (6) under the same conditions as Fig. 2. The numbers next to the lines in the figure indicate the actual range. The three lines are calculation results for the direction of the antenna beam center and the directions corresponding to the edges of the beam.

Since these two figures and the distance between the reception point and the summit of the mountain is about $3.7 \mathrm{~km}$, the echoes appeared in Fig. 1 is considered to be scattered signals from the mountain slope.

Note that despite the large HPBW of 20 degrees, the direction of the scattering object can be resolved with higher resolution using the Doppler frequency shift. 
$2017 / 04 / 28 \quad 02: 23: 12 \quad$ OKN_GPS SV\# 32 L2 az= $135.7 \mathrm{deg} \quad$ el= $67.0 \mathrm{deg}$

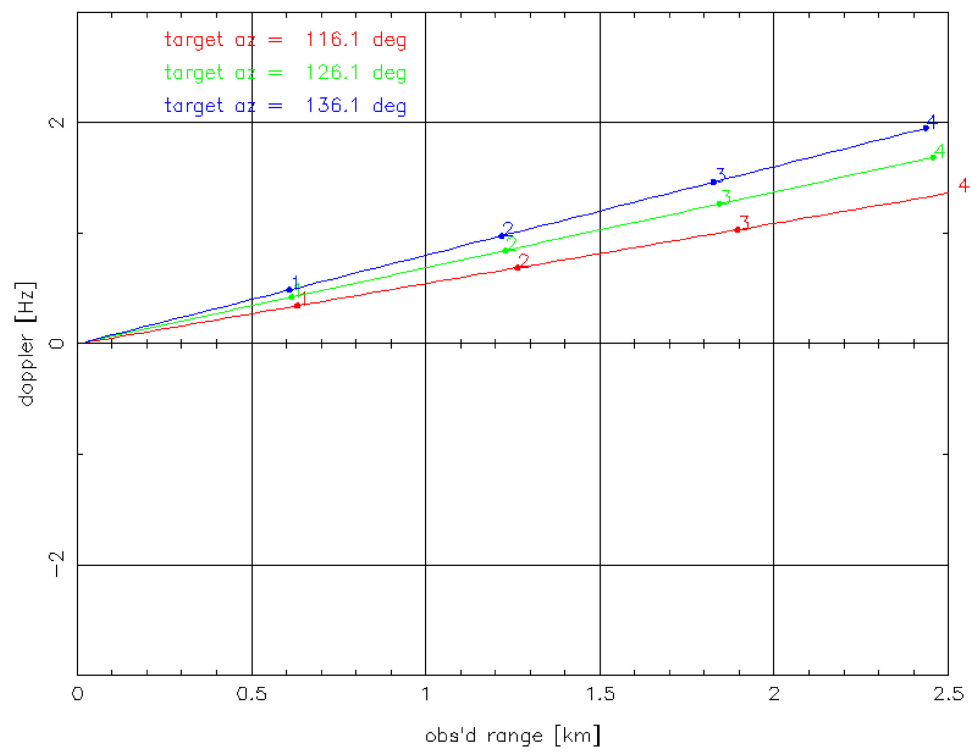

Fig. 3. Range-Doppler map calculated under the same conditions as the observation.

\section{Conclusion}

Using a beam antenna and long period integration, we successfully detected GPS signal scattered from the mountain $3 \mathrm{~km}$ away from the receiver. The Doppler frequency shift that increases in proportion to the range obtained in the experiment agrees well with the calculated value.

Since only the right-handed circularly polarization was observed in the experiment, we will also observe the left-handed circularly polarization to see the difference in echo due to the condition of the scattering surface.

Furthermore, there is a possibility to measure the anisotropy of the scattering surface by analyzing scatter of signals from satellites in different directions.

\section{Acknowledgments}

The authors appreciate that staffs of NICT VLBI group and Dr. Gotoh of NICT made us use the sampler control software they developed. 